\title{
Ultrasound-Guided Percutaneous Nephrostomy: How to Select Technique?
}

HUAN WANG, CHUANG CAO, CHANG-HUI WANG, XING-QIU FAN, JIANG-YA ZHONG

\begin{abstract}
Introduction: Ultrasound guided percutaneous nephrostomy (PCN) is a safe and effective treatment option for obstructive uropathy. Several techniques have been described in documents. However, the recommendation of PCN technique has not been proposed.
\end{abstract}

Aim: To determine the success and complication rates of ultrasound guided PCN. Meanwhile, we attempt to determine a baseline value in order to orient surgeon to select an optimal technique preoperatively.

Materials and Methods: This was a retrospective study conducted at Department of Urology of the First People's Hospital of Yueyang City, China. Total 150 PCNs under ultrasound guidance were analyzed. Two puncture techniques were used. In Seldinger technique (ST), ultrasound-guided puncture needle was inserted into the calyx; a drainage catheter was placed following the guidewire. In one-step technique (OT), the drainage catheter set was inserted directly into the collecting system under ultrasound guidance.

Statistical Analysis: A statistical analysis was performed using the SPSS software package (versions 19.0).
Results: Total 53 PCNs were performed using Seldinger technique, and $97 \mathrm{PCN}$ were performed using one-step technique. The ST group had higher technical success rate $(100 \%$ vs $87.6 \%, p=0.008)$. The ST group also obtained higher success rate in $<3 \mathrm{~cm}$ hydronephrosis subgroup ( $100 \%$ vs $44.4 \%, p<0.05)$. The success rate in $\geq 3 \mathrm{~cm}$ subgroup was similar ( $100 \%$ vs $97.5 \%$ ). The overall complication rate was $15.1 \%$ in ST group and $43.3 \%$ in OT group $(p<0.05)$. The major complication rate was $1.9 \%$ vs. $13.4 \%(p<0.05)$. The minor complication rate was $13.3 \%$ vs $29.9 \%(p<0.05)$.

Conclusion: Ultrasound-guided PCN is an effective and safe treatment method for obstructive uropathy. The Seldinger technique can effectively achieve success in all possible situations and is superior to the one-step technique when hydronephrosis is $<3 \mathrm{~cm}$. One-step PCN is an effective and safe procedure in selected patients with hydronephrosis $\geq 3 \mathrm{~cm}$. We recommend $3 \mathrm{~cm}$ of hydronephrosis should be an acceptable baseline for technique selection.

\section{Keywords: Grade, Hydronephrosis, Ureteral obstruction}

\section{INTRODUCTION}

Obstructive uropathy is defined as a condition occurring due to blockage of urine flow, resulting in increased pressure within the collecting system and destruction of the parenchyma of the kidney, in which there is loss of renal function. Once the diagnosis is suspected, treatment should be necessary to decompress. Currently available surgical drainage methods include operative nephrostomy, retrograde stenting and percutaneous nephrostomy (PCN) [1]. Operative nephrostomy or therapeutic nephrectomy may increase the morbidity and mortality. Treatment failure of retrograde ureteral stent is likely to occur. Moreover, sometimes a stent fails to alleviate the symptoms [2]. Therefore, PCN catheter placement recently has become a widely accepted alternative method.

Although PCN was developed using fluoroscopic guidance, ultrasound guided procedure is now safe, effective and reliable [3-5]. Two main PCN techniques have been described in documents $[6,7]$. The standard Seldinger technique is the most commonly used. The one-step technique (or direct puncture technique) may be an alternative option for the traditional technique in selected cases. Compared with the classic technique, the novel one-step technique has been reported to be a simple, less time consuming, less traumatic, and safe procedure $[8,9]$. 
Now the recommendation of PCN technique has not been proposed because of various available techniques and guidance imaging methods, special expertise requiring and different hydronephrosis grade associated with patients. Other main concerns are to improve primary success rate and minimize complication rate.

\section{AlM}

The aim of the study was to compare the success rate and the related complications of two different techniques. In addition, we attempt to determine a baseline value of hydronephrosis grade to orient surgeon's technique selection preoperatively.

\section{MATERIALS AND METHODS}

A retrospective study was carried out in 141 patients suffering from obstructive uropathy from January 2009 to January 2015. The study protocol was approved by the ethical committee of the First People's Hospital of Yueyang city, China.

All patients were subjected to routine investigations prior to the procedure, including blood count, chemistry and coagulation profile. Abdomen CT was done to decide the nature and site of ureteral obstruction. The patients were started on the empirical antibiotics when necessary. Those who simultaneously had untreated bleeding disorders were excluded from the study.

PCNs were performed for all patients entirely under ultrasound guidance. The procedure was explained to the patients and relatives. Informed consent was obtained from every patient.

The patients were placed on the ultrasound table in prone position. Ultrasound scanning (ACUSON Sequola 512 multicolor ultrasound instrument with transducer frequency $3.5 \mathrm{MHz}, \mathrm{USA}$ ) was performed using a transducer to obtain a transverse plane scan through the kidney to identify the degree of hydronephrosis. An anterior-posterior renal pelvis diameter was measured [Table/Fig-1]. The ideal puncture site into the kidney is via a posterior calyx approach. As soon as the initial puncture site was chosen, it was cleaned and draped. Local anesthesia was injected at the puncture site using $5 \sim 10 \mathrm{ml}$ of $2 \%$ lidocaine. We utilized one step drainage catheter set with safety string lock (Bioteque Corporation Taipei, Taiwan, China) as nephrostomy tract in all patients. The drainage catheter set includes a pigtail catheter (size from $7 \mathrm{Fr}$ to $16 \mathrm{Fr}$, length $30 \mathrm{~cm}$ ) and a trocar puncture needle.

\section{Procedure Technique}

In Seldinger technique, under real-time ultrasound guidance an 18G PTC needle (Hakko Co. Ltd, Nagano-ken, Japan) was inserted into the target calyx [Table/Fig-2]; Then a curved J tip 0.038-inch guide-wire (Cook, Bloomington, IN, USA) was passed through the needle sheath into collecting system with the help of an assistant. Then the sheath was removed and the wire was retained. The incision of skin and fascia was done. The drainage catheter set without inner metallic stylet was introduced into the collecting system following the guide-wire. The metallic trocar was removed. The drainage catheter was advanced into the collecting system further following the wire. Finally, after confirming the drainage catheter head curling in the collecting system, the guide wire was removed [Table/Fig-3]. The safety string lock was tightened. The catheter was secured to the skin using silk 4-0 and connected to a drainage bag.

In one-step technique, the skin and fascia were incised and then the scanning head was shifted over the incision to measure the distance between the skin and the calyx. The drainage catheter with puncture needle was inserted through the incision into the collecting system under realtime ultrasound guidance. Once the drainage set was at desired calyx for enough length, the trocar with the inner metallic tubes was removed and the drainage catheter was advanced into the collecting system further. No guide-wire and serial dilatation were undertaken during the procedure.

Urine samples were obtained and sent for Gram stain and culture. All patients ordered for having a rest in bed for 24 hours. Broad spectrum antibiotic and anti-hemorrhage drugs were routinely given for one day in post-procedure.

The patients were divided into Seldinger technique group (ST) and one-step technique group (OT) for this comparison study.

Procedure time was defined as the time between the first renal puncture to the completion of the procedure. Procedure success was defined as the drainage catheter into the collecting system.

\section{STATISTICAL ANALYSIS}

SPSS software package (versions 19.0, SPSS, Inc., Chicago, IL, USA) was used for all statistical analyses. Comparisons were made using Student's 't' tests and Pearson's chisquare tests. A p-value $<0.05$ was considered statistically significant.

\section{RESULTS}

A total of 141 patients were included in this analysis. Out of all nine patients required bilateral procedures. Therefore, total 150 renal units were performed PCNs. The patient's characteristics have been shown in [Table/Fig-4].

53 PCNs were performed using Seldinger technique. 97 PCNs were performed using one-step technique. Procedure characteristics were listed in [Table/Fig-5]. Totally, in OT group 

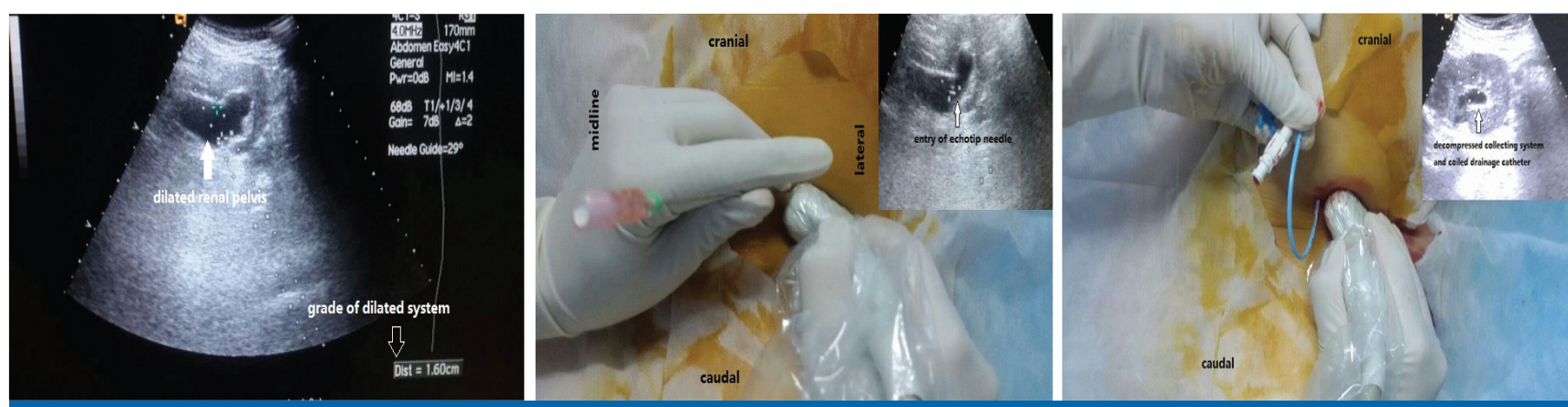

[Table/Fig-1]: Ultrasound scanning identified the renal pelvis was dilated to $1.6 \mathrm{~cm}$.

[Table/Fig-2]: Under real-time ultrasound guidance an 18G PTC needle was inserted into the renal pelvis

[Table/Fig-3]: Ultrasound scanning confirmed the drainage catheter head curling in the collecting system.

\begin{tabular}{|l|c|}
\hline Variable & Number of Patients \\
\hline Age (Year) (Range) & $50.5 \pm 11.7(25-79)$ \\
\hline Sex & 60 \\
\hline Male & 81 \\
\hline Female & \multicolumn{2}{|}{} \\
\hline Side & 62 \\
\hline Left & 79 \\
\hline Right & 9 \\
\hline Bilateral & 23 \\
\hline Disease Profile & 118 \\
\hline Malignant Disease & \\
\hline Urinary Calculi & \\
\hline [Table/Fig-4]: Patient's characteristics.
\end{tabular}

there were a lower rate in position change and intercostal puncture. In addition, in OT group there were less timeconsuming and larger nephrostomy tube utilized. But the most common tract size in both was $8 \mathrm{Fr}$. There were higher primary technique success rate in ST group than in OT group. 12 procedures failed in OT group, but successfully converted to the Seldinger technique. These 12 procedures were excluded from ST group for fear of observing complications. No insertion failure took place in ST group.

We further explored the correlation between the degree of collecting system dilation and primary technique success rate in both groups. The 150 kidney units were divided into two sub-groups taking $3 \mathrm{~cm}$ hydronephrosis grade as the critical value [Table/Fig-6]. The difference of the primary technique success rate was significant in less than $3 \mathrm{~cm}$ subgroup.

All patients tolerated the procedure well. There were 50 (33.3\%) complications encountered in the study. We assessed the complications followed the classification into major complication and minor complication [Table/Fig-5]. In terms of major complications and minor complications, significant difference was found in two groups. There was no death in both groups.

\begin{tabular}{|c|c|c|c|}
\hline & ST $(n=53)$ & OT $(n=97)$ & $\mathrm{p}$-value \\
\hline \multicolumn{4}{|l|}{ Position of procedure } \\
\hline Prone & $48(90.6 \%)$ & $96(99 \%)$ & 0.021 \\
\hline Lateral Decubitus Flank & $5(9.4 \%)$ & $1(1 \%)$ & \\
\hline \multicolumn{4}{|l|}{ Site of Puncture } \\
\hline Subcostal & $46(86.8 \%)$ & $94(96.9 \%)$ & 0.018 \\
\hline Intercostal & $7(13.2 \%)$ & $3(3.1 \%)$ & \\
\hline Mean procedure time (Min) & $15.6 \pm 4.3$ & $6.5 \pm 2.8$ & $<0.001$ \\
\hline Mean tract size $(\mathrm{Fr})$ & $8.0 \pm 0.7$ & $9.8 \pm 3.2$ & $<0.001$ \\
\hline $8 \mathrm{Fr}$ & $51(96.2 \%)$ & $63(64.9 \%)$ & \\
\hline$>8 \mathrm{Fr}$ & $2(3.8 \%)$ & $34(35.1 \%)$ & \\
\hline Procedure success rate & $53(100 \%)$ & $85(87.6 \%)$ & 0.008 \\
\hline Total of complications ${ }^{\star}$ & $8(15.1 \%)$ & $42(43.3 \%)$ & $<0.001$ \\
\hline Major complications & $1(1.9 \%)$ & $13(13.4 \%)$ & 0.020 \\
\hline Sepsis & 0 & $2(2.1 \%)$ & 0.293 \\
\hline Blood transfusion & 0 & $2(2.1 \%)$ & 0.293 \\
\hline Pleural injury & 0 & $2(2.1 \%)$ & 0.293 \\
\hline Renal pelvic injury & $1(1.9 \%)$ & $7(7.2 \%)$ & 0.165 \\
\hline Minor complications & $7(13.3 \%)$ & $29(29.9 \%)$ & 0.022 \\
\hline Pain need drugs & $3(5.7 \%)$ & $17(17.5 \%)$ & 0.041 \\
\hline Pneumonia & 0 & $1(1.0 \%)$ & 0.458 \\
\hline Tube blockage & $2(3.8 \%)$ & $6(6.2 \%)$ & 0.530 \\
\hline Fever with antipyretic & $2(3.8 \%)$ & $5(4.7 \%)$ & 0.701 \\
\hline
\end{tabular}

\begin{tabular}{|l|c|c|c|}
\hline $\begin{array}{l}\text { Degree of } \\
\text { Hydronephrosis }\end{array}$ & $\begin{array}{c}\text { Success Rate } \\
\text { of ST (\%) }\end{array}$ & $\begin{array}{c}\text { Success Rate } \\
\text { of OT (\%) }\end{array}$ & p-value \\
\hline$<3 \mathrm{~cm}(\mathrm{n}=50)$ & $32 / 32(100 \%)$ & $8 / 18(44.4 \%)$ & $<0.001$ \\
\hline$\geq 3 \mathrm{~cm}(\mathrm{n}=100)$ & $21 / 21(100 \%)$ & $77 / 79(97.5 \%)$ & 0.622 \\
\hline
\end{tabular}

[Table/Fig-6]: Comparison of success rate in varying degree of hydronephrosis.

\section{DISCUSSION}

Prompt drainage is indicated for obstructive uropathy if renal function is damaged, or obstruction is accompanied 
by acute infection, especially pyonephrosis. PCN catheters and cystoscopic insertion of a ureteral stent are preferred treatment choice. In the published literatures, most of studies announced that PCN group had a higher success rate, lower failure rate, less complication, shorter period of administration of antibiotics and better quality of life [10-13]. Therefore, many authors considered PCN a well-established procedure for this disease $[11,14]$.

In the early stage of PCN development, it was performed under fluoroscopic guidance. Currently, ultrasound has been used in lieu of fluoroscopy due to no radiation exposure and a higher success rate and lower complication rate. Other advantages ultrasound holds over X-ray include avoiding adjacent organ injury and intrarenal vascular injury, and offering a shortest access to the collecting system $[5,15]$. The advent of ultrasound imaging has allowed urologists for this procedure. In our center, this procedure has been considered the first-line treatment option for upper urinary obstruction, particularly with urgent pyronephrosis and poor renal function. However, certain limitations have to be considered such as non standardization of technical availability, special expertise always requiring and variability of technical success and complication.

Currently several techniques, such as Seldinger technique, onestep technique, one-stick technique, two-stick technique [16] and mixed technique [6], has been introduced for PCN. In our center, the most commonly used methods are Seldinger technique and one-step technique under solo ultrasound guidance. Among them, the Seldinger technique has been considered the golden standard for PCN. Other techniques evolved from it, such as one-stick technique and mixed technique. The classic method involves imaging guided puncture, guide wire insertion, serial dilatation and tube placement [17]. The success rate ranged from 84\% 100\% $[5,18]$. In the present study, we achieved a $100 \%$ success rate by using this approach. Serial dilatations were absent due to using the one step drainage catheter set. Hence, the present technique is more like one-stick technique and mixed technique. But a pyelography with contrast was performed in the above two techniques.

Compared with conventional technique, the one-step technique was considered to be easy, quick, safe and effective. Primary success rate ranged from 93\% to 96\% [8, $9,19]$. In the present study, the success rate of $87.6 \%$ was lower than the reported outcomes. Significant difference was found when compared with Seldinger group. But it was comparable to $80 \%$ $88 \%$ success rate of retrograde ureteral stent [10-12].

Now clear guidelines regarding technical selection for PCN have not been established. The clinical decision mostly depends on the urologist's preference and experience. Generally, Seldinger approach can be used in various degrees of hydronephrosis based on the published literatures. But the one-step approach has been attractive with an easy, quick and one-step process. Unfortunately, some studies demonstrated that this approach was indicated for moderate to severe hydronephrosis and not indicated for mild hydronephrosis [17]. In a comparative study including the Seldinger group and one-step group, the primary technical success rate was $98 \%$ vs 93\% [19]. In present study, there was significant difference in primary success rate of two techniques, $100 \%$ vs $87.6 \%$. Absence of hydronephrosis was the critical factor leading to puncture failure in one-step group. Therefore, it is absolutely necessary to establish a baseline of hydronephrosis for guiding technique selection in order to reduce the risk of fruitless reinsertion or surgical conversion. According to the findings in our study, we recommended $3 \mathrm{~cm}$ of collecting system dilatation should be an acceptable baseline.

Admittedly, whatever technique is selected, ultrasoundguided PCN is technically demanding. Study curve is long. Some special expertise is demanded for improving success rate. When Seldinger technique is used, identification of the puncture needle is a difficulty, especially in the early part of study. By using ultrasound probe puncture frame, the needle is more easily visualized. When one-step technique is used, overcoming the puncture resistance, which is associated with tube size, is a problem. To cut the skin deeply to latissimus dorsi layer and to enlarge the tissue using clamp may reduce the resistance. Smoothly and effortlessly introducing tube decreases the risk of injuring anterior calyx and pelvis. $8 \mathrm{Fr}$ tube is enough to reduce resistance and maintain free drainage.

The injury to major segmental branches of the renal vessels will cause severe bleeding. Color Doppler flow imaging demonstrates the intrarenal vessels and help manipulator to avoid the area of rich blood flow. It is important to follow the next steps for safe surgery: to visualize puncture needle, the target calyx and pelvis in the puncture path; to insert guidewire or tube under real-time ultrasound guidance.

Technically the procedure is performed more safely and easily in a dramatically dilated pelvicaliceal. PCN in a mild or nondilated pelvicaliceal system has been technically challenging. Following next steps may increase success rate. Firstly, ultrasound visualization of both pelvis and calyx throughout the procedure is the key to success [20]. Secondly, Seldinger approach is recommended as mentioned above. Thirdly, sometimes changing position to lateral may be useful for puncturing the ideal calyceal. Fourthly, once the puncture needle is inserted into the target calyx, introducing 
guide-wire into pelvis, not calyx, is demanded for avoiding collecting system collapse leading to tube placement failure. When necessary, 10 20ml normal saline should be injected slowly to dilate collecting system before guide-wire insertion. However, over distentsion should be forbidden in any situation, especially during acute infection, for fear of the increasing risk of sepsis by forcing infected urine into the venous system.

Complication associated with $\mathrm{PCN}$ is an important issue requiring concern. In the published studies, overall complication rate of about $30 \%$ has been reported $[6,20]$, major complications, such as injury to adjacent organs, severe bleeding required transfusion, and sepsis are as high as $6 \sim 7 \%$, whereas minor complications may be seen in up to $28 \%$, which is comparable to our study , $9.3 \%$ and $24 \%$ respectively. Mortality related to the procedure has less been reported. It is equally distributed among ultrasound guided and fluoroscopic guided groups. In a series reported by Naeem $\mathrm{M}$ et al, Ahamad I et al., and Jalbani $\mathrm{MH}$ et al., respectively, a risk of sepsis was $2.0 \%, 3.5 \%$ and $7.5 \%[1,20,21]$. In the setting of pyonephrosis, septic shock is reported to occur in $7 \%$ to $9 \%$ [22]. The rate of hemorrhage requiring transfusion with $\mathrm{PCN}$ was reported to be between $1 \%$ and $4 \%$ [23]. In the present study, the incident rate of sepsis, transfusion and adjacent organ injury was 1.3\%(2/150), 1.3\%(2/150) and $6.7 \%(10 / 150)$ respectively. It was accordance with the reported results. Post-PCN tract blockage or dislodgment observed ranged from $4 \%-37 \%$ while it was $5.3 \%(8 / 150)$ in the present study. No dislodgment took place. Careful suturing and advancement of the catheter well into the pelvis will minimize the risk of dislodgement.

It is not clear whether different technical application may affect the complication occurrence. In Wah TM's study, the major complications was $4.1 \%$ vs. $3.2 \%$, the minor complication rate was $5 \%$ vs. $1.3 \%$, and tube complications, such as drainage catheter dislodgement and blockage were 29.5\% vs. $17.7 \%$,in Seldinger group and one-step group [19]. In the present study, significant difference was found in overall complication rate (15.1\% vs. $43.3 \%)$, major complication rate $(1.9 \%$ vs. $13.4 \%)$ and minor complication rate $(13.3 \%$ vs. $29.9 \%$ ). However, we noted that a statistically significant difference only occurred in the specific complication of post-procedure pain. We can not ascertain that Seldinger technique is associated with lower complication rate due to small samples. But our findings supported that a small tube could reduce pain in the immediate postoperative time.

\section{LIMITATIONS}

There are some limitations in the present study. As a comparative study the number of cases is relatively small. Since special expertise is needed for ultrasound guided
PCN, the unskilled surgeon may have adverse impact on the observation of outcome.

\section{CONCLUSION}

Ultrasound-guided PCN is an effective and safe treatment method for obstructive uropathy. The Seldinger technique can effectively achieve success in all possible situations and is superior to the one-step technique when hydronephrosis is $<3 \mathrm{~cm}$. One-step PCN is an effective and safe procedure in selected patients with hydronephrosis $\geq 3 \mathrm{~cm}$. We recommend $3 \mathrm{~cm}$ of hydronephrosis should be an acceptable baseline for technique selection.

\section{REFERENCES}

[1] Naeem M, Jan MA, Ullah A, Ali L, Khan S, Amin-ul-Haq, et al. Percutaneous nephrostomy for relief of upper urinary tract obstruction: an experience with 200 cases. JPMI. 2010;24:14752.

[2] Richter S, Ringel AA, Shalev M, Nissenkorm I. The indwelling ureteric stent: a "friendly" procedure with unfriendly high morbidity. BJU Int. 2009; 85:408-11.

[3] Martino P. Ultrasound-guided percutaneous nephrostomy. Arch Ital Urol Androl. 2000;72:324-27.

[4] Baishya RK, Dhawan DR, Jagtap J, Sabnis R, Desai MR. Percutaneous nephrostomy under ultrasound guidance. Indian J Nephrol. 2011;21: 67.

[5] Mahmood T, Younus R, Ahmad F, Memon S, Moavia A. Ultrasound as a reliable guidance system for percutanous nephrostomy. J Coll Physician Surg Pak. 2007;17:15-18.

[6] Agostini S, Dedola GL, Gabbrielli S, Masi A. A new percutaneous nephrostomy technique in the treatment of obstructive uropathy. Radiol Med. 2003;105:454-61.

[7] Montvilas P, Solvig J Johansen TE. Single-centre review of radiologically guided percutaneous nephrostomy using "mixed" technique: success and complication rates. Eur $J$ Radiol. 2011;80:553-58.

[8] Lodh B, Gupta S, Singh AK, Sinam RS. Ultrasound guided direct percutaneous nephrostomy (PCN) tube placement: stepwise report of a new technique with its safety and efficacy evaluation. J Clin Diagn Res. 2014; 8: 84-87.

[9] Karim R, Sengupta S, Samanta S, Aich RK, Das U, Deb P. Percutaneous nephrostomy by direct puncture technique: An observational study. Indian J Nephrol. 2010; 20: 84-88.

[10] Danilovic A, Antonopoulos IM, Mesquita JL, Lucon AM. Likelihood of retrograde double-J stenting according to ureteral obstructing pathology. Int Braz J Urol. 2005;31:431-36.

[11] Mokhmalji H, Braun PM, Martinez Portillo FJ, Siegsmund M, Alken P, Köhrmann KU. Percutaneous nephrostomy versus ureteral stents for diversion of hydronephrosis caused by stones: a prospective, randomized clinical trial. J Urol. 2001;165:108892.

[12] Wenzler DL, Kim SP, Rosevear HM, Faerber GJ, Roberts WW, Wolf JS Jr. Success of ureteral stents for intrinsic ureteral obstruction. J Endourol. 2008; 22: 295-99.

[13] Yagci C, UstunerE, Atman ED, Baltaci S, Uzun C, Akyar S. Diuretic agent and normal saline infusion technique for ultrasoundguided percutaneous nephrostomies in nondilated pelvicaliceal systems. Cardiovasc Intervent Radiol. 2013;36:492-97.

[14] Goldsmith ZG, Oredein-McCoy O, Gerber L, Bañez LL, Sopko $\mathrm{DR}$, Miller MJ, et al. Emergent ureteric stent vs percutaneous 
nephrostomy for obstructive urolithiasis with sepsis: patterns of use and outcomes from a 15-year experience. BJU Int. 2013;112:122-28.

[15] Osman M, Wendt-Nordahl G, Heger K, Michel MS, Alken P, Knoll T. Percutaneous nephrolithotomy with ultrasonographyguided renal access: experience from over 300 cases. BJU Int 2005; 96:875-78.

[16] Dagli M, Ramchandani P. Percutaneous nephrostomy: technica aspects and indications. Semin Intervent Radio. 2011; 28:424-37.

[17] Carrafiello G, Laganà D, Mangini M. Complications of percutaneous nephrostomy in the treatment of malignant ureteral obstructions: single-centre review. Radiol. Med. 2006; 111:562-71.

[18] Sood G, Sood A, Jindal A, Verma DK, Dhiman DS. Ultrasound guided percutaneous nephrostomy for obstructive uropathy in benign and malignant diseases. Int Braz J Urol. 2006; 32:281-86.
[19] Wah TM, Weston MJ, Irving HC. Percutaneous nephrostomy insertion: outcome data from a prosptective multi-operator study at a UK training centre. Clin Radiol. 2004; 59:255-61.

[20] Ahmad I, Saeed Pansotal M, Tariq M, Shahzad Saleem M, Ali Tabassum S, Hussain A. Comparison between double J(DJ) ureteral stenting and percutaneous nephrostomy (PCN) in obstructive uropathy. Pak J Med Sci. 2013;29:725-29.

[21] Janlbani MH, Deenari RA, Dholia KR. Role of Percutaneous nephrostomy (PCN) in malignant ureteral obstruction. J Pak Med Assoc. 2010; 60:280-83.

[22] Ramchandani P, Cardella JF, Grassi CJ, Roberts AC, Sacks D, Schwartzberg MS. et al. Quality improvement guidelines for percutaneous nephrostomy. J Vasc Interv Radiol. 2003;14:S277-81.

[23] Ramchandani P, Cardella JF, Grassi CJ, Roberts AC, Sacks D, Schwartzberg MS,et al. Quality improvement guidelines for percutaneous nephrostomy. J Vasc Interv Radiol. 2001;12:1247-51.

\section{AUTHOR(S):}

1. Dr. Huan Wang

2. Dr. Chuang Cao

3. Dr. Chang-hui Wang

4. Dr. Xing-qiu Fan

5. Dr. Jiang-ya Zhong

\section{PARTICULARS OF CONTRIBUTORS:}

1. Associate Professor, Department of Urology, the People's Hospital of Yueyang City, Yueyang, Hunan, P.R. China.

2. Associate Professor, Department of Ultrosonography, the People's Hospital of Yueyang City; Yueyang, Hunan, P.R. China.

3. Resident, Department of Urology, the People's Hospital of Yueyang City, Yueyang, Hunan, P.R. China.
4. Professor, Department of Urology, the People's Hospital of Yueyang City, Yueyang, Hunan, P.R. China.

5. Attending Doctor, Department of Urology, the People's Hospital of Yueyang City, Yueyang, Hunan, P.R. China.

NAME, ADDRESS, E-MAIL ID OF THE CORRESPONDING AUTHOR:

Dr. Huan Wang,

No. 39, Dongmaoling Road,

Yueyang Tower District, Yueyang City, Hunan Province, P.R.-414000, China.

E-mail: Wanghuan20087615@hotmail.com

FINANCIAL OR OTHER COMPETING INTERESTS:

None.

Date of Publishing: Apr 01, 2016 